This is a pre-print of an article published in

International Journal of Modern Physics A, 35 (2020) 2050135

The final authenticated version is available online at:

https://doi.org/10.1142/S0217751X20501353

\title{
Lorentz transformation of a charge-current density and "relativistic polarization" of a moving current loop
}

\author{
Alexander Kholmetskii \\ Department of Physics, Belarusian State University, 4, Nezavisimosti Avenue, \\ 220030 Minsk, Belarus, alkholmetskii@gmail.com \\ Oleg Missevitch \\ Institute for Nuclear Problems, Belarusian State University, 11, Bobruyskaya str., \\ 220030 Minsk, Belarus \\ Tolga Yarman \\ Okan University, Akfirat, Istanbul, Turkey
}

\begin{abstract}
We show that the claim by Franklin (Int. J. Mod. Phys. A 35, 2050061 (2020)) with respect to the vanishing charge distribution over the perimeter of an electrically neutral moving current loop is erroneous and is based on a misinterpretation of physical meaning of Lorentz transformations. Moreover, we show that the development of non-vanishing electric dipole moment by a moving current loop (which we named as "relativistic polarization") represents a direct implication of covariant formulation of classical electrodynamics of material media. In this respect, we analyze some subtle effects related to the motion of magnetic dipoles in an electromagnetic field and disclose their physical meaning.
\end{abstract}

\section{Introduction}

In a recent paper [1], Franklin claimed that the Lorentz transformation of a charge-current density four-vector $j^{\mu}=[\rho(\boldsymbol{r}, t), \boldsymbol{j}(\boldsymbol{r}, t)]$ was seriously misinterpreted to the moment, and (in his opinion) relativistic polarization of an electrically neutral moving current loop does not exist. (Hereinafter, we follow the designations of [1] in units $c=1$ ). In order to specify this claim, the author [1] presented the Lorentz transformation between the frame of observation $S^{\prime}$ and the proper frame $S$ of a uniformly moving neutral current loop $(\rho=0)$ in the case, where the vectors $\boldsymbol{j}$ and $\boldsymbol{V}$ are collinear to each other:

$\rho^{\prime}(\boldsymbol{r}, t)=\gamma \boldsymbol{V} \cdot \boldsymbol{j}(\boldsymbol{r}, t)$,

$\boldsymbol{j}^{\prime}(\boldsymbol{r}, t)=\gamma \mathrm{j}(\boldsymbol{r}, t)$,

Then, he pointed out that for the actual determination of the charge distribution in the moving loop, one has to transform further the space-time four-vector $(t, r)$ from $S$ to $S^{\prime}$, in order to present $\rho^{\prime}, \boldsymbol{j}^{\prime}$ as functions of $t^{\prime}, \boldsymbol{r}^{\prime}$. Having implemented such an additional Lorentz transformation for space-time coordinates, Franklin finally concluded that the charge density of conduction electrons, as the function of $t^{\prime}$, remains equal to the change density of positive 
ions in the crystal cells, so that the net charge density in the laboratory frame $S^{\prime}$ remains equal to zero (see eq. (6) of ref. [1].

However, in section 2 we disclose the erroneous character of physical interpretation of eqs. (1), (2) by Franklin, and totally invalidate all subsequent results and conclusions made in ref. [1]. Concurrently, we discuss physical meaning of the "relativistic polarization" of a moving magnetic dipole through continuity equation for carries of current written for different inertial observers and also clarify some subtle points of macroscopic classical electrodynamics, which we believe are helpful for better understanding of its fundamental basis and relativistic consistency. Finally, we conclude in section 3.

\section{Charge distribution in a moving current loop and relativistic transformation of electric (magnetic) dipole moment}

We remind that in ref. [1], Franklin applied transformations (1), (2) to an electrically neutral moving current loop of a rectangular form, assuming that one of its segments (a "sampling cell" in the terminology of [1]) is collinear to the velocity $\boldsymbol{V}$ of the loop, as is measured in the laboratory frame $S^{\prime}$. Then, determining the charge density in this segment for a laboratory observer, Franklin claimed that the actual charge density $\rho^{\prime}$ for the considered segment should be determined as the function of laboratory time $t^{\prime}$, which thus, according to him, requires to carry out the additional Lorentz transformation for space-time coordinates between $S^{\prime}$ and $S$; moreover, he claimed that the need to carry out this additional transformation has been overlooked by Panofsky and Phillips in their classical book [2], as well as their followers (see [1], refs. 1-14). Thus, implementing this additional transformation, and keeping the constancy of $t$ in the calculation of total charge of the "sampling cell" in the frame S', Franklin finally finds that the total charge density of the sampling cell turns to be equal to zero (see eq. (6) of [1]).

We will show that this conclusion by Franklin is erroneous, while the standard relativistic result - the development of an electric dipole moment by a moving magnetic dipole, in our case, the electrically neutral current loop - remains to be correct.

The core mistake by Franklin is related to his misinterpretation of the special relativity principle, which, as is well known, establishes the possibility to describe any phenomenon in any inertial reference frames realizable in Nature. This implies that the Lorentz transformation of any four-vector $A^{\mu}$ defined in an inertial frame $\mathrm{S}$ (i.e., $A^{\mu}=A^{\mu}(\boldsymbol{r}, t), \mu=0 \ldots 3$ ) to another inertial frame $S^{\prime}$ yields the four-vector $A^{\prime \mu}$, which, in the analysis of its properties in this frame, should be considered already as the function of $\boldsymbol{r}^{\prime}, t^{\prime}$ i.e.

$A^{\prime \mu}\left(\boldsymbol{r}^{\prime}, t^{\prime}\right)=A^{\prime \mu}\left(\boldsymbol{r}\left(\boldsymbol{r}^{\prime}, t^{\prime}\right), t\left(\boldsymbol{r}^{\prime}, t^{\prime}\right)\right)$.

From the physical viewpoint, eq. (3) signifies that an observer in $S^{\prime}$ finds sufficient to investigate the properties of the four-vector $A^{\prime \mu}$ as the function of his proper space-time coordinates $\boldsymbol{r}^{\prime}, t^{\prime}$, and no longer addressing to the frame $\mathrm{S}$.

Thus, according to the general rule (3), the Lorentz transformations for the components of four-current density in a conducting current loop should be written in the form

$$
\begin{aligned}
& \rho^{\prime}\left(\boldsymbol{r}^{\prime}, t^{\prime}\right)=\gamma \boldsymbol{V} \cdot \boldsymbol{j}\left(\boldsymbol{r}\left(\boldsymbol{r}^{\prime}, t^{\prime}\right), t\left(\boldsymbol{r}^{\prime}, t^{\prime}\right)\right), \\
& \boldsymbol{j}^{\prime}\left(\boldsymbol{r}^{\prime}, t^{\prime}\right)=\gamma \boldsymbol{j}\left(\boldsymbol{r}\left(\boldsymbol{r}^{\prime}, t^{\prime}\right), t\left(\boldsymbol{r}^{\prime}, t^{\prime}\right)\right)
\end{aligned}
$$

at $\boldsymbol{j}$ being collinear to $\boldsymbol{V}$. Here, the charge density $\rho^{\prime}$ and current density $\boldsymbol{j}^{\prime}$ in the frame $S^{\prime}$ are expressed as functions of $r^{\prime}, t^{\prime}$, so that in the analysis of the "sampling cell" by Franklin, we have to calculate its charges at fixed time $t^{\prime}$ of the frame $\mathrm{S}^{\prime}$, but not at fixed time $t$ of the frame $\mathrm{S}$, as Franklin erroneously supposed in eq. (3) of ref. [1].

This result invalidates the conclusions of ref. [1], and confirms the validity of the standard relativistic approach by Panofsky and Phillips [2] for the calculation of charge distribution over the perimeter of a moving current loop, as measured in the laboratory frame $\mathrm{S}^{\prime}$. 
At the same time, the majority of books on classical electrodynamics (e.g., [2-4]) do not suggest any specific physical mechanisms for the explanation of eq. (4); for example, Panofsky and Phillips [2] present only some general argumentation toward the explanation of this equation via the relativity of simultaneity of events for different inertial observers. This argumentation is fully correct, but, at the same time, it is too general. For this reason, it does not elucidate the physical mechanism of this effect - i.e., the development of the electric dipole moment by a moving magnetic dipole - which we propose to name as "relativistic polarization".

An attempt to understand the mechanism of the "relativistic polarization" of a moving current loop has been made in ref. [5] (not mentioned in [1]) on the basis of the continuity equation for the conduction electrons of the loop, which should be valid both in the rest frame $\mathrm{S}$ of the loop and in the laboratory frame $\mathrm{S}^{\prime}$. In this approach, the "relativistic polarization" of the loop can be understood already at a qualitative level, if we introduce into consideration the cross-sectional areas $A_{i}$ of the wire for each segment of the rectangular loop $(i=1 \ldots 4)$, comoving with the loop, and then realize that a number of conduction electrons $N_{e i}$ passing across each area $A_{i}$ during a unit time interval $t_{u}$ (corresponding to the electric charge $e N_{e i}$ and the current $I_{i}=e N_{e i} / t_{u}$ ) should be the same for each segment due to the requirement of continuity of the current flaw in both frames $S$ and $S^{\prime}$ (see Fig. 1). At the same time, the path covered by the conduction electrons during the unit time interval, is different in the frame $S^{\prime}$ for the lower and upper segments of the loop due to the Einstein law of velocity composition. Therefore, in the frame $S^{\prime}$, the charge density of conduction electrons in the lower $\left(\rho_{e 1}^{\prime}\right)$ and upper $\left(\rho_{e 3}^{\prime}\right)$ segments of the loop should be different from each other, in order to keep the equality of currents in both segments.

In particular, for the lower segment 1 , we have in the frame $S^{\prime}$ (below we abandon the units with $c=1$ ):

$e N_{1}^{\prime}=\rho_{e 1}^{\prime} A_{1}^{\prime} t_{u}^{\prime}\left(\frac{u+V}{1+u V / c^{2}}-v\right)=\rho_{e 1}^{\prime} A_{1}^{\prime} t_{u}^{\prime}\left(\frac{u \sqrt{1-V^{2} / c^{2}}}{1+u V / c^{2}}\right)$,

whereas for the upper segment we analogously derive,

$$
e N_{3}^{\prime}=\rho_{e 3}^{\prime} A_{3}^{\prime} t_{u}^{\prime}\left(\frac{u \sqrt{1-V^{2} / c^{2}}}{1-u V / c^{2}}\right) \text {. }
$$

Thus, comparing eqs. (6) and (7), we find that the requirement of continuity $N_{1}^{\prime}=N_{3}^{\prime}$ is realized only under the inequality

$\rho_{e 1}^{\prime} \neq \rho_{e 3}^{\prime}$,

which thus once again invalidates the results of [1].

In a similar way, for the side segments 2,4 of the loop, we straightforwardly find $\rho_{e 2}^{\prime}=\rho_{e 4}^{\prime}$.

In order to determine the total charge density in all segments of the loop in the explicit form, in ref. [5] we have used the requirements of continuity of current for four corners of the loop, and arrived at the Lorentz transformation (4) for charge density in all segments of the loop.

The obtained results indicate a close relationship between the Lorentz transformation for the four-current density, and the requirement of continuity of the flow of charges in a circuit, as is seen by different inertial observers.

In our opinion, this mechanism, being based on the continuity equation for carries of current (which also serves as the relativistic generalization of the first Kirchhoff law for electric circuits [5]), clarifies in a better way the physical meaning of "relativistic polarization" of a moving current loop, than the general statement by Panofsky and Phillips [2] about relativi- 
ty of simultaneity of events for different inertial observers. At the same time, one should stress the full compatibility of both approaches [2,5] towards eqs. (4), (5); in particular, the Einstein law of velocity composition used in the derivation of eqs. (6), (7) is essentially based on the relativity of simultaneity of events for different inertial observers emphasized by Panofsky and Phillips [2].

In this respect, we find erroneous the claim by Franklin [1] that the major part of previous papers on the subject (including 14 papers in the reference list of [1]) "...just implemented (without question) the Panofsky and Phillips result. None of them have anything like an independent derivation of eq. (1)". Now we are inclined to think that this claim stems from an incomplete knowledge of Franklin about the available literature on the subject, e.g., about ref. [5], where eq. (1) is derived via the continuity equation for the conduction electrons in a closed circuit - the way, not mentioned in ref. [2].

Further on, we point out that a current loop can be considered as a magnetic dipole with the proper magnetic dipole moment $\boldsymbol{m}=\boldsymbol{F} I$, where $\boldsymbol{F}$ is the area of the loop, and $I$ is the current of the loop in its rest frame S. Thus, the effect of the "relativistic polarization" of a moving current loop means that it acquires the electric dipole moment

$$
\boldsymbol{p}^{\prime}=\frac{\boldsymbol{v} \times \boldsymbol{m}}{c^{2}}
$$

in the laboratory frame $S^{\prime}$. Eq. (10) represents a direct consequence of covariant formulation of classical electrodynamics of material media, where the transformation of magnetizationpolarization tensor and its further integration over the volume of a compact dipole yields the following relationships [2]:

$$
\begin{gathered}
\boldsymbol{p}^{\prime}=\boldsymbol{p}-\frac{(\gamma-1)}{\gamma^{2}}(\boldsymbol{p} \cdot \boldsymbol{v}) \boldsymbol{v}+\frac{\boldsymbol{v} \times \boldsymbol{m}}{c}, \\
\boldsymbol{m}^{\prime}=\boldsymbol{m}-\frac{(\gamma-1)}{\gamma v^{2}}(\boldsymbol{m} \cdot \boldsymbol{v}) \boldsymbol{v}+\frac{\boldsymbol{p} \times \boldsymbol{v}}{c},
\end{gathered}
$$

where $\gamma=\left(1-v^{2} / c^{2}\right)^{-1 / 2}$ is the Lorentz factor. The last term on rhs of eq. (11) is responsible for the "relativistic polarization" of a moving magnetic dipole (see eq. (10)), while the physical meaning of other terms in eqs. (11), (12) is discussed in details in ref. [2].

Next, it is worth to mention the paper by Fisher [6], where the relativistic transformation of the magnetic dipole moment is found in the form

$$
\boldsymbol{m}^{\prime}=\frac{\boldsymbol{m}}{\boldsymbol{\gamma}}-\frac{(\gamma-1)}{2(\gamma+1) c^{2}} \boldsymbol{v} \times(\boldsymbol{v} \times \boldsymbol{m})+\frac{\boldsymbol{p} \times \boldsymbol{v}}{2 c},
$$

which obviously disagrees with the standard equation (12).

Commenting this result by Fischer, Franklin notices that the derivation of relativistic transformation of the magnetic dipole moment in ref. [6] "...does use the moving frame time variable $t^{\prime}$, but makes the mistake to holding it fixed". However, the first step by Fischer is correct, and does agree with the fact that the Lorentz transformation for any four-vector $A^{\mu}(\boldsymbol{r}$, $t$ ) from an inertial frame $S$ to another inertial frame $S^{\prime}$ yields the four-vector $A^{\prime \mu}\left(\boldsymbol{r}^{\prime}, t^{\prime}\right)$ already expressed via the primed space-time coordinates, as we have mentioned above.

The actual error made by Fischer in the derivation of eq. (13) is disclosed in ref. [7], and it represents the incorrect extension of the known definition of the proper magnetic dipole moment

$\boldsymbol{m}=\frac{1}{2} \int(\boldsymbol{r} \times \boldsymbol{j}) d \Omega$

(being valid in the rest frame of the dipole $S$, where $\Omega$ is the volume of the dipole) to an arbitrary inertial frame of observation $S^{\prime}$, wherein the dipole is moving. Indeed, considering any inertial frame of observation $S^{\prime}$, wherein the dipole is moving with a finite velocity $\boldsymbol{V}$, one 
should realize that the Lorentz transformation between the frames $S$ and $S^{\prime}$ for the current density $\boldsymbol{j}^{\prime}$, i.e.,

$$
\boldsymbol{j}^{\prime}=\boldsymbol{j}+\frac{(\gamma-1)}{V^{2}}(\boldsymbol{j} \cdot \boldsymbol{V}) \boldsymbol{V}+\gamma \boldsymbol{V} \rho
$$

is valid for the total current density, which thus includes not only the contribution of conduction electrons $\boldsymbol{j}_{\text {el }}^{\prime}$, but also contains the density of convective current of ions $\boldsymbol{j}_{\text {ions }}^{\prime}=\rho_{\text {ions }} \boldsymbol{v}$ moving along with the dipole. At the same time, it is obvious that $\boldsymbol{j}_{\text {ions }}^{\prime}$ does not contribute to the magnetic dipole moment $\boldsymbol{m}^{\prime}$ measured in the frame $\mathrm{S}^{\prime}$. Hence, the generalization of the definition (14) to the inertial frame $S^{\prime}$ adopted by Fischer, i.e.

$\boldsymbol{m}^{\prime}=\frac{1}{2} \int\left(\boldsymbol{r}^{\prime} \times \boldsymbol{j}^{\prime}\right) d \Omega^{\prime}$,

where $\boldsymbol{j}^{\prime}$ obeys the Lorentz transformation (15), is incorrect, insofar as it includes the contribution of convective current of ions, not affecting the magnetic dipole moment. Therefore, the substitution of eq. (15) into eq. (16), leading to the transformation by Fischer (13), is fundamentally incorrect from the physical viewpoint.

As we have shown in ref. [7], the correct extension of eq. (14) to an arbitrary inertial frame $S^{\prime}$ is given in the form

$\boldsymbol{m}^{\prime}=\frac{1}{2}\left(\int \boldsymbol{r}^{\prime} \times \boldsymbol{j}_{e l}^{\prime}\right) d \Omega^{\prime}$,

where the contribution of convective current of positive ions of a moving loop is excluded. Further on, having applied the Lorentz transformation between $\boldsymbol{r}$ and $\boldsymbol{r}^{\prime}$, and determined the relativistic transformation for the current density of conduction electrons $\boldsymbol{j}_{e l}$ between the frames $S$ and $S^{\prime}$, we arrived at the common transformation (12) for the magnetic dipole moment [7].

This result one more confirms the correctness of the standard covariant formulation of classical electrodynamics in material media and relativistic transformation of the electric and magnetic dipole moments (11), (12), according to which a moving magnetic dipole acquires the electric dipole moment (10).

The effect of "relativistic polarization" of a moving magnetic dipole (10) gave rise to the known paradox by Mansuripur, according to which the electric component of Lorentz force develops a torque on the moving magnetic dipole due to co-moving charge, whereas in the rest frame of the dipole and charge, such a torque is absent [8].

The available comments on the Mansuripur paradox (refs. 6-13 of [1]) were classified by Franklin as "conflicting". However, this is well understandable, because the Lorentz invariant expression for the force on a dipole, which consistently resolves all relativistic paradoxes related to the motion of electric (magnetic) dipoles in an electromagnetic field (see ref. [9]), has been found few years later since publication of the letter by Mansuripur [8].

\section{Conclusion}

We have shown that the conclusion by Franklin with respect to the vanishing electric dipole moment of a moving current loop is based on a misinterpretation of the Lorentz transformations, and should be denied. To the contrary, the moving magnetic dipole actually develops the electric dipole moment, and the physical meaning of this effect can be understood with the continuity equation for carries of current written for different inertial observers. This and other results presented above confirm full consistency of modern covariant formulation of macroscopic classical electrodynamics, and allows us to understand better a number of its physical implications. 


\section{References}

1. J. Franklin, Int. J. Mod. Phys. A 35, 2050061 (2020).

2. W. K. H. Panofsky and M. Phillips, M Classical Electricity and Magnetism, 2nd edn. (Addison-Wesley, 1962).

3. W. G. V. Rosser, An Introduction to the Theory of Relativity (Butterworths, London, 1964).

4. J. D. Jackson, Classical Electrodynamics, 3rd edn. (Wiley, New York, 1998).

5. A. L. Kholmetskii and T. Yarman, Eur. J. Phys. 31, 1233 (2010).

6. G. P. Fisher, Am. J. Phys. 39, 1528 (1971).

7. A. L. Kholmetskii, O.V. Missevitch and T. Yarman, Eur. Phys. J. Plus 31, 1233 (2010).

8. M. Mansuripur, Phys. Rev. Lett. 108, 193901 (2012).

9. A. L. Kholmetskii, O.V. Missevitch and T. Yarman, Ann. Phys. 369, 139 (2016). 


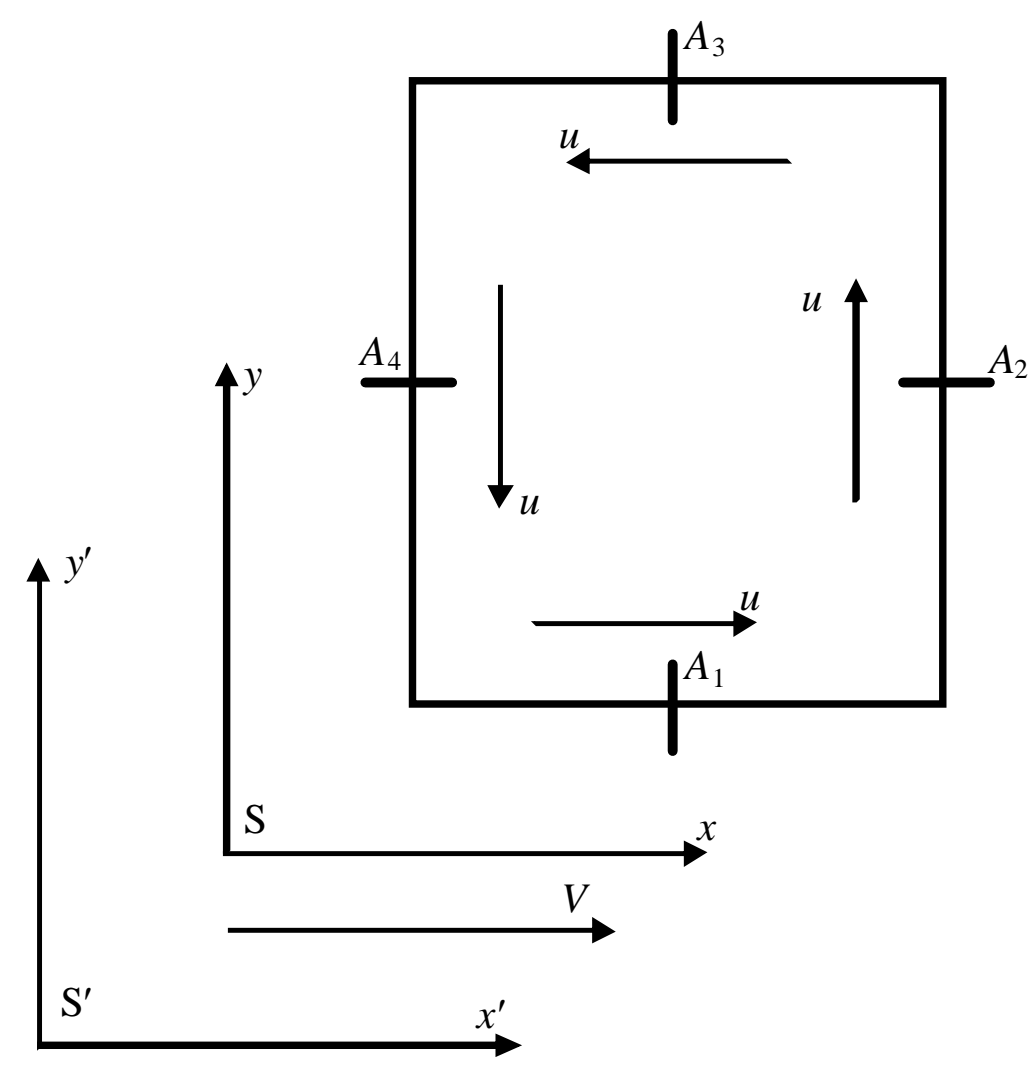

Fig. 1. Rectangular current loop lying in the $x y$-plane moves at constant velocity $V$ along the $x$-axis of the laboratory frame $\mathrm{S}^{\prime}$. On each segment of the loop, we designate the cross-sectional areas $A_{1} \ldots A_{4}$ and emphasize that the number of conduction electrons passing across each $A_{i}$ during a unit time (i.e., the current in the circuit) should be the same for observers in both frames $\mathrm{S}$ and $\mathrm{S}^{\prime}$. Here $u$ stands for the flow velocity of conduction electrons in the rest frame of the loop $\mathrm{S}$. 